\title{
Qualifikation und Konfession am Beispiel München: Kirchenzugehörigkeit in Münchner Chefetagen
}

\author{
ROBERT GEIPEL
}

Ludwig-Maximilians-Universität München, Institut für Geographie

\section{Abstract}

\section{Hierarchies of qualification and denomination: the example of Munich}

The article gives a short overview on previous engagements of the author in the field of the geography of religion. Then it starts out to ascertain that confessional denomination can be used as an indicator for selective mobility of highly qualified people. The faculties of the two Munich universities as well as civil servants of Munich's public administration (23.000 cases), employees of major companies in industry (BMW and DASA), commercial services like "Munich Re-Insurance" and the media (Bavarian Broadcasting Company), with altogether 37.000 cases, were included in the study. It shows that the higher the rank in the various hierarchies the bigger is the share of Protestants (who are in a diaspora situation here) and of those who have left their denominations. In this group, disintegration from traditional relations in favour of an individualistic lifestyle is evident.

Key words: religious denomination - qualification - Munich - geography of religion

Meine Beschäftigung mit Fragen der Religionsgeographie geht über fast 50 Jahre zurück:

- Begonnen hatte es in Frankfurt 1952 mit einer Dissertation bei Wolfgang Hartke über eine evangelische Enklave, das „Wallauer Ländchen“ inmitten des kurmainzer Katholizismus unter dem Titel „Soziale Struktur und Einheitsbewusstsein als Grundlagen geographischer Gliederung"(GEIPEL 1952).

- Das Thema Konfessionen wurde in den 60er und 70er Jahren weitergeführt mit Untersuchungen an 53.000 Abiturienten in Hessen (später auch in Rheinland-Pfalz) aus zehn Jahrgängen über unterschiedliche Bildungsbeteiligung in evangelischen und katholischen Gebieten (GEIPEL 1965, siehe Tab. 1).

- In Bayern befasste ich mich in den 90er Jahren mit Innovationsdiffusion am Beispiel der Kirchenaustrittsbewegung, die vom großen urbanen Zentrum München in Oberbayern in die Diaspora ausstrahlte und sich an der Peripherie der protestantischen Kernlande in Franken abschwächte (GEIPEL 1996). 
Tab. 1 Konfessionelle Zugehörigkeit der Gesamtbevölkerung und der Schüler an Gymnasien in Hessen in Prozent

\begin{tabular}{|l|c|c|}
\hline & evangelisch & katholisch \\
\hline Gesamtbevölkerung (16.6.1961) & 63 & 32 \\
\hline Schüler öffentlicher Gymnasien & 71,3 & 26 \\
\hline Schüler privater Gymnasien & 46 & 51,4 \\
\hline Schüler an Gymnasien insgesamt & 67,7 & 29,7 \\
\hline Abiturienten 1955-1964 insgesamt & 68,6 & 27,8 \\
\hline
\end{tabular}

Zuletzt beschäftigte ich mich mit dem Thema „Konfession und Qualifikation“ am Beispiel der Kirchenzugehörigkeit in Münchner Verwaltungen und Betrieben. Es gibt zu solchen sehr privaten Daten keinen direkten Zugang. Es wäre völlig unmöglich, sie durch Befragungen zu ermitteln. Wenn trotzdem Aussagen über 37.000 Personen vorgelegt werden können, dann bedingt durch den Umstand, dass die Arbeitgeber die Lohnsteuerkarten verwalten, auf denen vermerkt ist, an welche Religionsgemeinschaften die Kirchensteuer abgeführt wird. Es gibt dabei nur drei Kategorien: die Hauptkonfessionen evangelisch und katholisch sowie die Kategorie „Sonstige/ohne“. Unter „Sonstigen“ werden kleinere Religionsgemeinschaften, aber auch orthodoxe Christen und Moslems verstanden, während die Bezeichnung „ohne" wahrscheinlich vor allem Kirchenaustritte vermuten lässt. Es ist klar, dass diese drei Parameter nur den höchst nüchternen Befund über eine noch vorhandene oder nicht mehr vorhandene, wie auch immer geartete kirchliche Bindung abbilden, wie sie sich aus dem Umstand ergibt, dass Kirchensteuer entrichtet wird.

Nun sind die Belegschaften der sieben ausgewählten Betriebe und Dienststellen nach Gehaltsstufen und Dienstbezeichnungen hierarchisch gegliedert. Es gibt zum Beispiel ein unteres, mittleres und oberes Management oder (bei der Stadtverwaltung Münchens) Besoldungs- und BAT-Tarifstufen, die von A5 bis A16 und B-Stellen von BAT X bis BAT I reichen. Die Universitäten unterscheiden verschiedene Professorengruppen (C2, C3) bis hin zu den Ordinarien (C4). In jeder dieser Kategorien befinden sich jeweils so viele Personen, dass die Anonymität gewährleistet war und sich keinerlei Datenschutzprobleme ergaben.

Es besteht schon lange Gewissheit darüber, dass Diasporagruppen in ihrer anderskonfessionellen Umgebung, also zum Beispiel die Hugenotten in Frankreich, aber auch die sogenannten „Nordlichter" in der katholischen Umgebung Altbayerns, einen besonderen Aufstiegswillen bekunden. Häufig nehmen sie bessere Berufspositionen als die Altansässigen ein. Sie haben ja bereits durch ihre Mobilitätsbereitschaft, das heißt, ihre Entscheidung, die angestammte Heimat zu verlassen und in die Fremde zu ziehen, Mut und Entschlusskraft bewiesen. Da sie nicht so stark in der Landwirtschaft, im Haus-, Grund- und Geschäftsbesitz verankert sind wie die Einheimischen, setzen sie eher auf die Karte Bildung, weshalb ihre Kinder überproportional häufig weiterführende Schulen und Universitäten besuchen. Die Rolle der Franken, die Übersiedlung der Siemens-Spitze von Berlin nach München, der Zustrom aus der früheren DDR hat das Element der Evangelischen in 
München verstärkt, und es erschien verlockend festzustellen, in welchen Positionen des Arbeitslebens sie sich denn nun nach einer solchen Wanderung wiederfinden.

Bei der wohl „münchnerischsten“ Untersuchungseinheit, den 23.000 Beamten und Angestellten der Münchner Stadtverwaltung (Abb. 1), bietet sich folgendes Bild: Auf der Ebene der mittleren Beamten und Angestellten ist die Zahl der Evangelischen am geringsten. In der höchsten Besoldungsstufe (B) aber hat sich ihr Anteil fast verdoppelt, und der Anteil der „Sonstigen“ ist mit fast $36 \%$ fast so hoch wie jener der Katholiken. Stellen wir der bodenständigen Stadtverwaltung die mobilen Führungsschichten von zwei Weltunternehmen gegenüber: BMW und DASA. Bei den mehr als dreitausend Führungskräften von BMW in der Zentrale München steigt der Anteil der „Sonstigen“ von 44 über 50 zu fast $54 \%$, während jener der Katholiken von 38 auf $28 \%$ fällt und sich jener der Evangelischen unerheblich steigert (Abb. 2). Ein forschungsintensiver global player wie BMW unterscheidet sich also, je statushöher umso stärker, von der konfessionellen Normalverteilung seines Standorts. Das ist noch ausgeprägter am Teilstandort der rund 1.400 bei der Satellitenforschung Beschäftigten der DASA der Fall (Abb. 3), wo der Anteil der Katholiken fast spiegelbildlich von 40 auf $30 \%$ sinkt, während jener der Evangelischen von 24 auf $40 \%$ steigt.

Auch an den beiden Münchner Universitäten gibt es bei den unteren Besoldungsstellen bis zum Amtmann (A3 bis A12) weit überwiegend Katholiken, dann holen die Evangelischen bei den Professorenstellen auf, und bei den C3-Stellen der TU sind sogar die Konfessionslosen und die Evangelischen im Jahr 2000 jeweils stärker vertreten als ihre katholischen Kollegen (Abb. 4). Bei einer früheren Untersuchung aus dem Jahre 1985 war dies noch viel ausgeprägter der Fall. Hier überwogen an beiden Universitäten bei den Ordinarien (C4) die evangelischen Kollegen die Katholiken (GeIPeL 1989, siehe Abb. 5). Das Rekrutierungsgebiet bei der Besetzung von Professorenstellen an Münchner Universitäten reichte damals weiter in den Westen und Norden Deutschlands, während man sich heute auch bei den bayerischen Universitätsneugründungen bedienen kann. Bei beiden Untersuchungen stammen die meisten Angehörigen der unteren Dienststufen aus dem heimischen Arbeitskräftepotential, die höheren kommen von weiter her.

Beim Bayerischen Rundfunk mit seinen fast 6.000 festangestellten Mitarbeitern insgesamt ist mit 40,7\% die Gruppe der „Sonstigen“ am größten, gefolgt von den Katholiken (Abb. 6). Bei den Anlernberufen, Auszubildenden und Volontären sind die Katholiken am stärksten vertreten, es gibt die wenigsten Ausgetretenen, wohl weil der erste Gehaltszettelschock noch nicht eingetreten ist, der vielen aus Steuervermeidungsgründen den Kirchenaustritt nahe legt. Bei den künstlerischen Berufen wie dem Chor und den Orchestern überwiegen die den Kirchen Fernstehenden.

Bei anderen Betrieben sind ähnliche Beobachtungen zu machen. Als Fazit lässt sich feststellen: Nimmt man sämtliche 37.000 Mitarbeiter der sieben von uns untersuchten Einrichtungen zusammen, so ergibt die Auswertung der konfessionsbezogenen Meldungen noch ein ähnliches Bild wie das der Gesamtbevölkerung 
(Tab. 2). Untersucht man aber nur die rund 1000 absoluten Spitzenpositionen (die "Chefetagen"), so verschiebt sich dieses Bild zugunsten der Evangelischen und der Sonstigen. Die Evangelischen gewinnen zehn, die Sonstigen 1,3 Prozent, bei den Katholiken fehlen elf Prozent. Damit bestätigt sich unsere Vermutung von der selektiven Wanderung der Höherqualifizierten an einen Ort, an dem sie ihre Qualifikation am besten in Wert setzen können. Das sind diejenigen Städte, die eine Konzentration von Firmenleitungen, Schulen, Hochschulen und Forschungsabteilungen anbieten können, also Wachstumszentren von hohem Freizeit- und Prestigewert wie München.

In solchen Städten verändern sich die Arbeitsverhältnisse laufend, und es stellen sich dabei Trends heraus, die für die Entwicklung der Arbeitswelt typisch sind. Für München lässt sich folgender Trend festhalten:

In den zehn Jahren zwischen 1989 und 1998 hat die Zahl der sozialversicherungspflichtig Beschäftigten um mehr als 40.000 abgenommen und ging vom Höchststand von 705.000 im Jahre 1992 auf 633.000 zurück. Dieser Rückgang traf aber die einzelnen Wirtschaftsabteilungen sehr unterschiedlich. So nahmen die Zahlen im produzierenden Gewerbe von 225.000 auf 166.000 um rund $59.000 \mathrm{ab}$ ( $-26 \%)$, während sie bei den Dienstleistungen von 188.000 auf 232.000 um 44.000 zunahmen (+23\%). Typisch hierfür ist die stark konjunkturabhängige Entwicklung im Baugewerbe, das von 36.000 Beschäftigten in einem Jahrzehnt auf $22.000 \mathrm{zu}-$ rückging $(-37 \%)$. Auch die Veränderungen bei der sozialen Schichtung sind bemerkenswert. Die Zahl der Arbeiter sank von 233.000 auf 178.000 (-23\%), jene der Angestellten stieg hingegen um 14.000 von 440.000 auf 454.000. Die Relation der Geschlechter hat sich dabei kaum verändert.

Tab. 2 Konfessionelle Zusammensetzung (in Prozent) der Einwohner Münchens sowie der Mitarbeiter und der Spitzenpositionen aller untersuchter Großbetriebe 1999/2000

\begin{tabular}{|l|c|c|c|}
\hline & katholisch & evangelisch & Sonstige \\
\hline Einwohner & 45,4 & 16,3 & 38,3 \\
\hline Sämtliche Mitarbeiter & 46,5 & 17,5 & 36,0 \\
\hline Spitzenpositionen & 35,2 & 27,5 & 37,3 \\
\hline $\begin{array}{l}\text { Überschuss/Defizit Spitzenpositionen/ } \\
\text { Mitarbeiter }\end{array}$ & $-11,3$ & $+10,0$ & $+1,3$ \\
\hline
\end{tabular}

Die Jahresberichte des Arbeitsamtes München sprechen eine ähnlich deutliche Sprache:

In den fünf Jahren zwischen 1992 und 1997 verminderte sich die Zahl der dort erfassten sozialversicherungspflichtig Beschäftigten um rund 60.000. Dabei gibt es typische Verlierer und Gewinner, die hier einmal in wenigen ausgewählten Berufsgruppen in ihren Eckdaten gegenübergestellt seien (Tab. 3).

Die dem Menschen zugewandten ersten vier aufgeführten Berufsgruppen werden stärker, die vier letzten, die einer rationalisierbaren technischen Welt angehören, nehmen ab, am deutlichsten die Drucker. Ingenieure, Chemiker, Physiker und 
Informatiker bleiben gleich. Die hier ausgewählten beiden konträren Berufsgruppen machen mit (1992) 22,3\% und (1997) 23,6\% immerhin rund ein Fünftel aller Beschäftigten aus, so dass die beschriebene Tendenz auf relativ hohen Aussagewerten beruht. Innerhalb dieser nur fünf Jahre stiegen die Angehörigen des ersten Blocks um rund 15.000 (14,6\%), die des zweiten sanken um 16.000 (14,8\%). Im ersten Block überwiegen die Angestellten mit (1997) 96\%, im zweiten Block (außer den Technikern) die Arbeiter mit 88\%. Somit bestätigt unsere Analyse den allgemeinen Trend zur Angestelltengesellschaft. Diese Angestelltengesellschaft ist gekennzeichnet durch die zunehmende Ablösung ihrer Mitglieder von traditionellen Bindungen an Stand, Partei, oder Gewerkschaft. Die Angestellten sind die Schrittmacher bei der Veränderung von Verhaltensstilen und werden immer wieder als Auslöser von Modernisierungsschüben benannt. Deshalb sind sie auch bei den Kirchenaustritten weit überproportional vertreten. Dies wurde bereits bei der Analyse der Konfessionszugehörigkeit der Führungskräfte bei BMW erwähnt, wo die Zahl der „Sonstigen“ bei den oberen Führungskräften im Jahre 1999 bereits 53,6\% betrug. Da relativ wenige Evangelische in München die typischen Berufe der Alteingesessenen ausüben, finden wir sie nur schwach unter den Arbeitern und Handwerkern, aber gerade bei den Angestellten stark vertreten. Von ihnen wurde gerade behauptet, dass sie besonders zu Kirchenaustritten neigen. Bei der letzten Volkszählung, jener von 1987, waren zwar nur 35\% aller bayerischen Erwerbstätigen Angestellte. Von den im Jahre 1991 Ausgetretenen waren das aber 55\%.

Eine ähnliche Verzerrung gilt beim Alter: Während die 18-50jährigen zusammen knapp die Hälfte der 1987 Gezählten ausmachten, waren es bei den 1991 Ausgetretenen $83 \%$. Es sind also die Jüngeren und Besserverdienenden, welche in diesen Jahren die Kirche verlassen haben.

Aus diesen Beobachtungen lässt sich ein erstes Fazit ziehen:

Die beschriebenen Trends in der Arbeitswelt Münchens und unsere Befunde über sieben Betriebe und Dienststellen weisen über die lokalen Befunde hinaus. Sie dokumentieren, dass mit sozialem Aufstieg in Form höherer Positionen in Behörde oder Firma die ,amtliche“, das heißt steueraktenkundige Religiosität abnimmt.

Zwar verbietet es sich, gesicherte Aussagen über die Ursachen der Korrelation zwischen Kirchenferne und beruflicher Position zu machen. Aber es lässt sich sehr wohl behaupten, dass sich die berufliche Mobilität und die religiöse Mobilität (in Form einer Entfernung von der Kirche) im Wechselspiel verstärken. Dabei dürfte es sich bei den untersuchten Personen wohl mehrheitlich um einen persönlichen Kirchenaustritt im Laufe ihrer Biographie handeln, und wohl nur ein kleiner Teil von ihnen ist gar nicht getauft worden.

Der Berufsstatus ist - zusammen mit Bildung und Einkommen - eine Variable, welche den sozialen Status eines Individuums abbildet.

Soziale Ungleichheitsstrukturen, die früher erhebliche Bedeutung hatten wie die Gliederung in Stände, Klassen oder soziale Schichten, lösen sich zunehmend zugunsten einer Gliederung in Lebensstilgruppierungen auf, hinter denen eine in- 
dividuelle Entscheidung für diesen oder jenen Lebensstil steht. Die früher eher schicksalhafte Zuordnung zu gesellschaftlichen Milieus (das „Arbeitermilieu“, das ,katholische Milieu“) ist zunehmend einer vom Ich selbst getroffenen Wahl eines bestimmten Lebensstils gewichen. In der Nachkriegszeit hat auch die durch Geburt bestimmte Zuordnung zu konfessionellen Gruppierungen an Bedeutung verloren.

Tab. 3 Veränderungen in der Berufsgruppenstruktur Münchens zwischen 1992 und 1997 (ausgewählte Berufsgruppen)

\begin{tabular}{|l|r|r|r|c|}
\hline Berufsgruppe & \multicolumn{1}{|c|}{$\mathbf{1 9 9 2}$} & $\mathbf{1 9 9 7}$ & Tendenz & In Prozent \\
\hline Künstler & 9.748 & 10.190 & +442 & +4 \\
\hline Publizisten, Dolmetscher, Bibliothekare & 7.702 & 8.286 & +584 & +7 \\
\hline Gesundheitsdienste & 45.578 & 52.880 & +7.302 & +16 \\
\hline Sozial- und Erziehungsberufe & 38.722 & 45.243 & +6.621 & +17 \\
\hline Ingenieure, Chemiker, Physiker, Informatiker & 46.322 & 46.076 & -246 & +-0 \\
\hline Drucker & 9.250 & 6.428 & -2.822 & -30 \\
\hline Chemiearbeiter & 6.272 & 5.192 & -1.080 & -17 \\
\hline Schlosser, Mech. & 51.585 & 42.609 & -8.976 & -17 \\
\hline Techniker & 42.949 & 39.733 & -3.216 & -7 \\
\hline Insgesamt & 950.511 & 890.983 & -59.528 & -6 \\
\hline
\end{tabular}

Vor diesem Hintergrund ist der hohe Anteil der „Sonstigen“ bei den Angaben zur Kirchensteuer als ein starker Indikator für die Auflösung schicksalhafter Zuordnung zugunsten individueller Wahlen der Lebensführung anzusehen. Die Religionsgemeinschaft stiftete, bei aller tendenziellen Auflösung traditioneller Strukturen der Gesellschaft, noch lange Zeit zumindest im geistig-moralischen Bereich „Gemeinschaft". Heute zeigt sich, dass die individuelle Bestimmung des Lebensstils auch auf diesem Gebiet weit fortgeschritten ist, und dass es vor allem die höheren Statusgruppen sind, bei denen sich diese Lebensstilwahl am stärksten ausgebreitet hat. Wer aber sorgt anstelle der Kirchen für den sozialen Kitt unter unseren Eliten? Was haben die Kirchen in den Chefetagen noch zu sagen?

Mein zweites Fazit lautet:

Seit Max Weber wurden Zusammenhänge zwischen Theologie und Ökonomie deutlich. Die hier referierten Untersuchungen weisen in die Richtung Konfessionen und Machtstrukturen.

Die „Political Correctness“ hindert uns häufig daran, solche Fragen zu stellen. Wenn wir bei den Überlegungen zur zukünftigen Ausrichtung religionsgeographischer Forschung vorankommen wollen, sollten wir aber an dem Problem von Konfession und Machtausübung nicht vorbeigehen. 


\section{Literatur}

GEIPEL, R. (1952): Soziale Struktur und Einheitsbewusstsein als Grundlage geographischer Gliederung. Dargestellt am Beispiel des „Ländchens“ zwischen Frankfurt und Wiesbaden. Rhein-Mainische Forschungen, 38 .

ders. 1965: Sozialräumliche Strukturen des Bildungswesens. Studien zur Bildungsökonomie und zur Frage der gymnasialen Standorte in Hessen. Frankfurt a.M.: Diesterweg.

ders.1968: Bildungsplanung und Raumordnung. Studien zur Standortplanung von Bildungseinrichtungen und zu räumlichen Aspekten des Bildungsverhaltens in Hessen. Frankfurt a.M.: Diesterweg.

ders. 1989: Evangelische in München - ein Indikator für sozialräumliche Prozesse. In: HEYER, R. \& HOMMEL, M. (Hrsg.): Stadt und Kulturraum. Peter Schöller zum Gedenken (= Bochumer Geogr. Arb., Bd. 50), 48-63.

ders. 1996: Evangelische in Bayern - ein Indikator für sozialräumliche Prozesse. Zeitschrift für Bayerische Kirchengeschichte, 65, 105-141.

ders. 1998: Die empirische Basis für kirchliche Arbeit in München. In: Theologischer Ausschuss der Dekanatssynode München - Evangelisches Forum München (Hrsg.): Evangelisch für München. Dokumentation einer Tagung und Sozialstruktureller Atlas der Evangelischen Kirchengemeinden Münchens, 25-43. München.

ders. 2000: Qualifikationshierarchien und Konfessionen am Beispiel Münchens. In: Beiträge zur Hochschulforschung, Heft 1-2, 2000, 169-189.

GEIPEL, R., P. GEISSER (1998): Sozialstruktureller Atlas der Evangelischen Kirchengemeinden in München. In: Theologischer Ausschuss der Dekanatssynode München - Evangelisches Forum München (Hrsg.): Evangelisch für München. Dokumentation einer Tagung und Sozialstruktureller Atlas der Evangelischen Kirchengemeinden Münchens, 71-82. München.

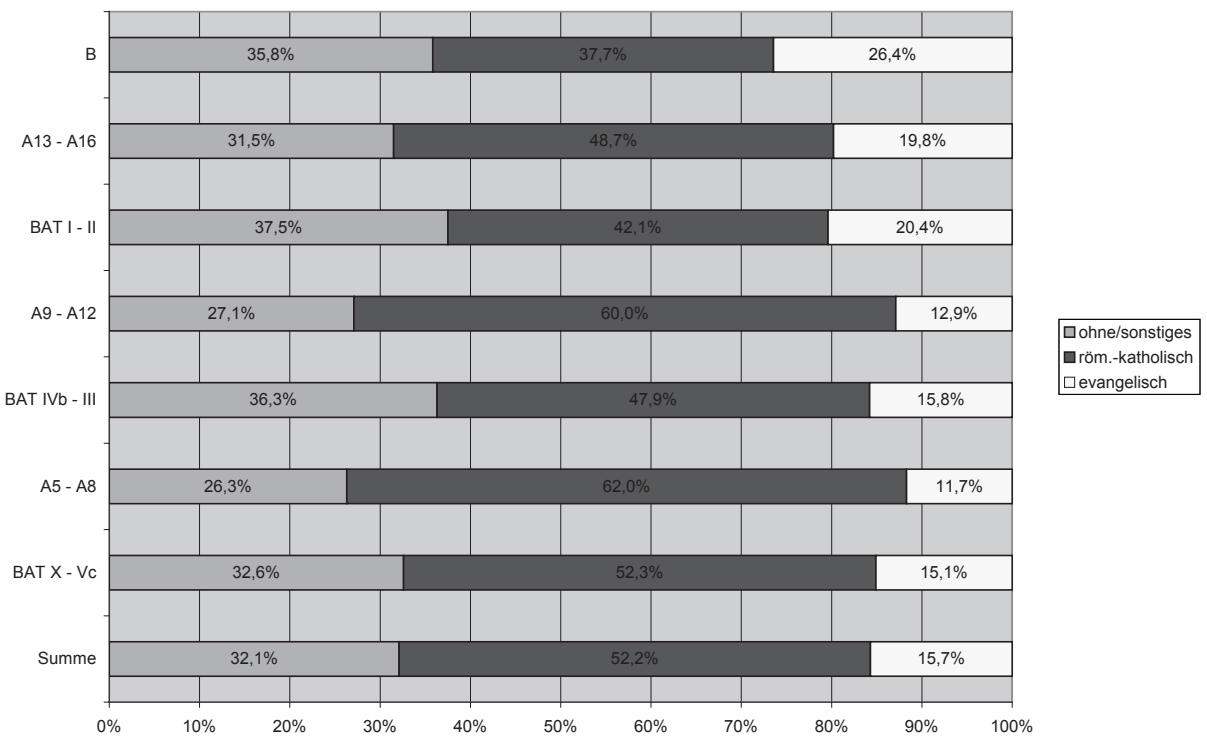

Abb. 1 Besoldung/Vergütung und Konfessionen in der Stadtverwaltung Münchens, 1999 


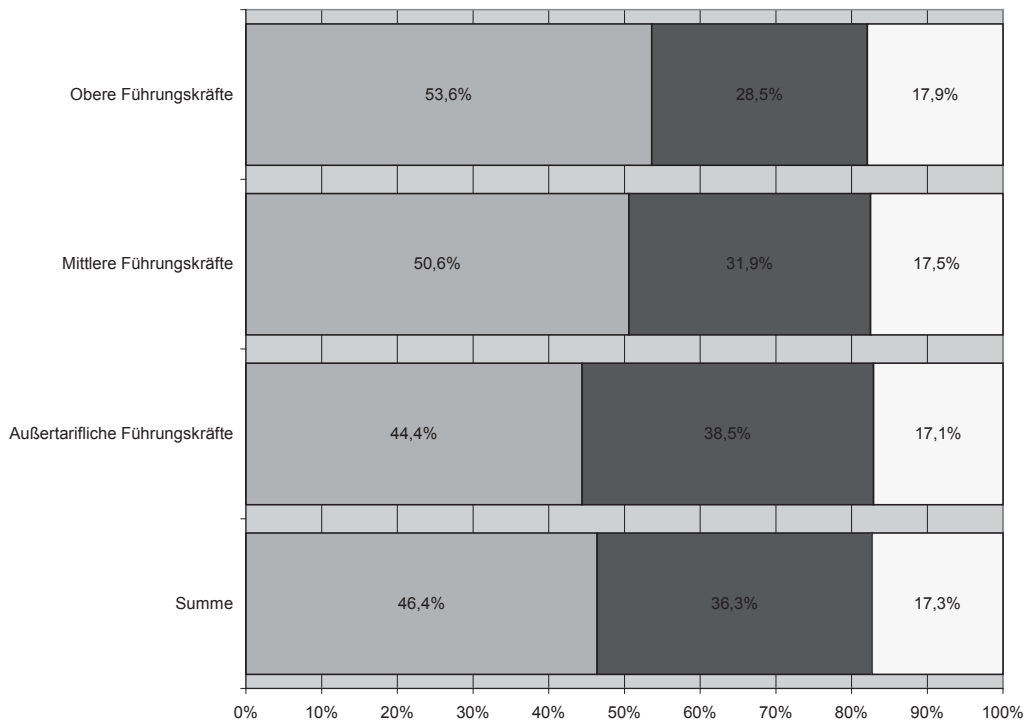

Abb. 2 Konfessionszugehörigkeit der Führungskräfte bei BMW (München), 1999

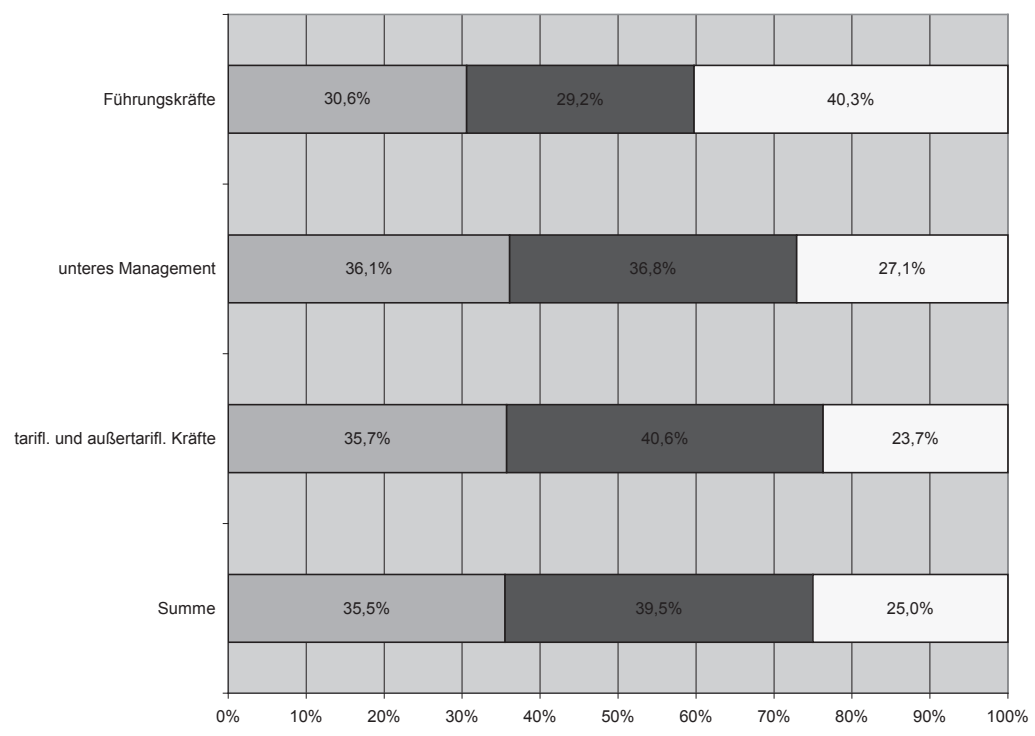

Abb. 3 Qualifikationsstufen und Konfessionszugehörigkeit bei der DASA, 2000 


\section{LMU}

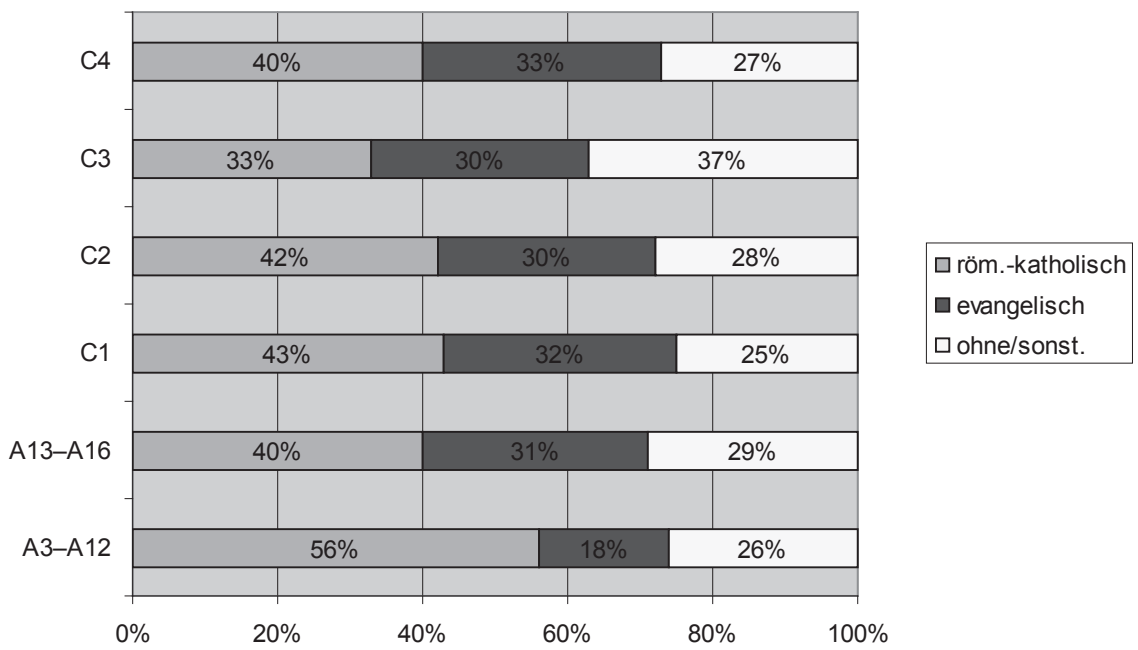

TU

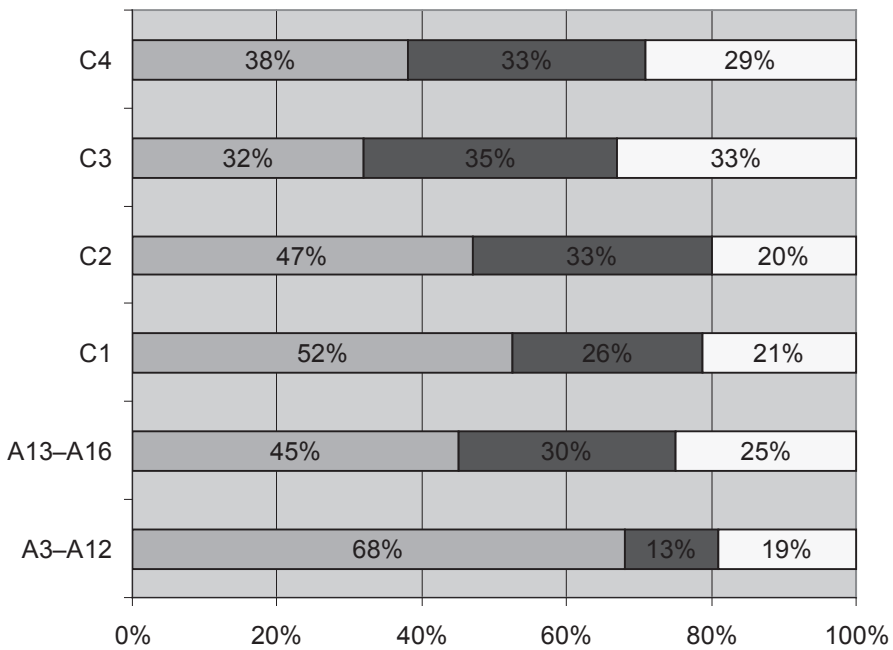

$\square$ röm.-katholisch

$\square$ evangelisch

$\square$ ohne/sonst.

Abb. 4 Kirchensteuerstatistik über die Besoldungsgruppen an der Ludwig-Maximilians-Universität (LMU) und an der Technischen Universität (TU) im Jahr 2000 


\section{LMU}

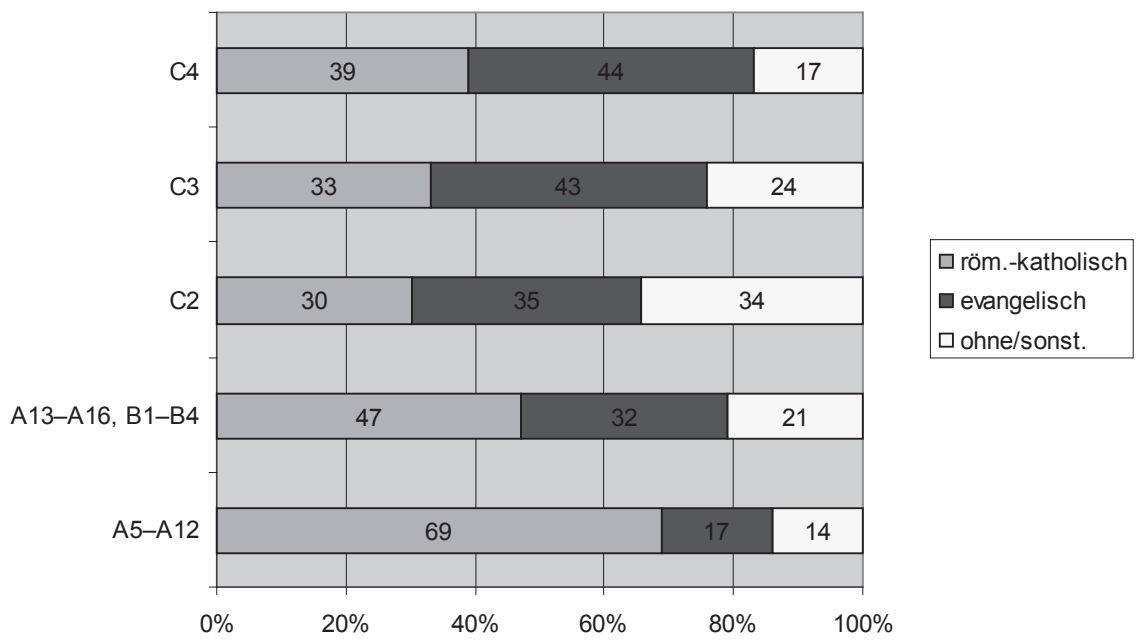

TU

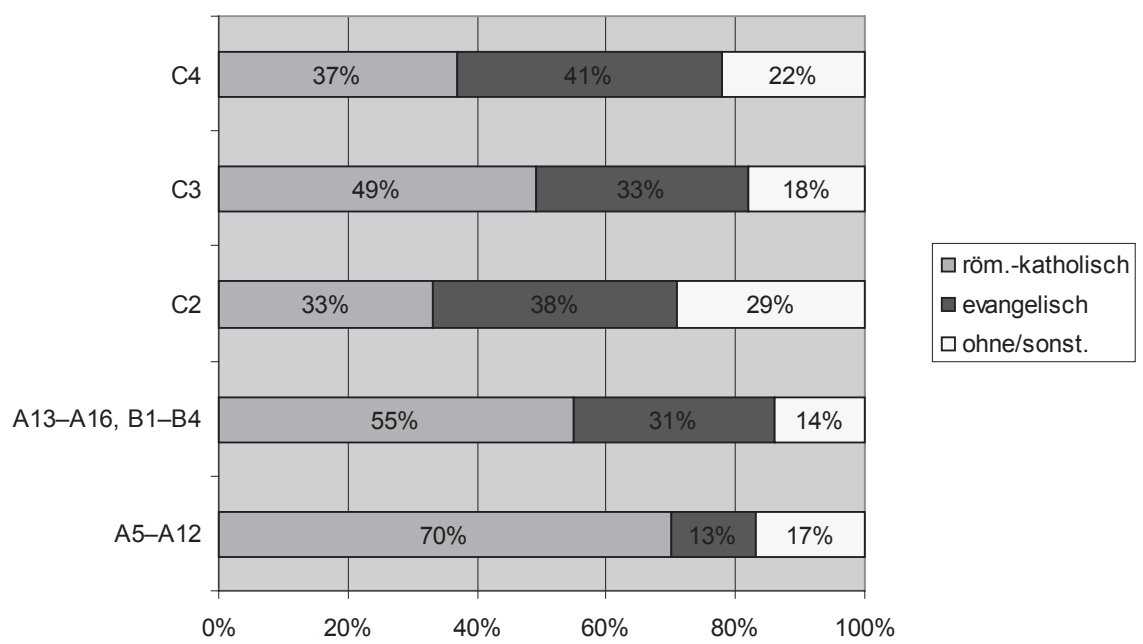

Abb. 5 Kirchensteuerstatistik über die Besoldungsgruppen an der Ludwig-Maximilians-Universität (LMU) und an der Technischen Universität (TU) 1985 (Quelle: GeIPeL 1989, 61) 


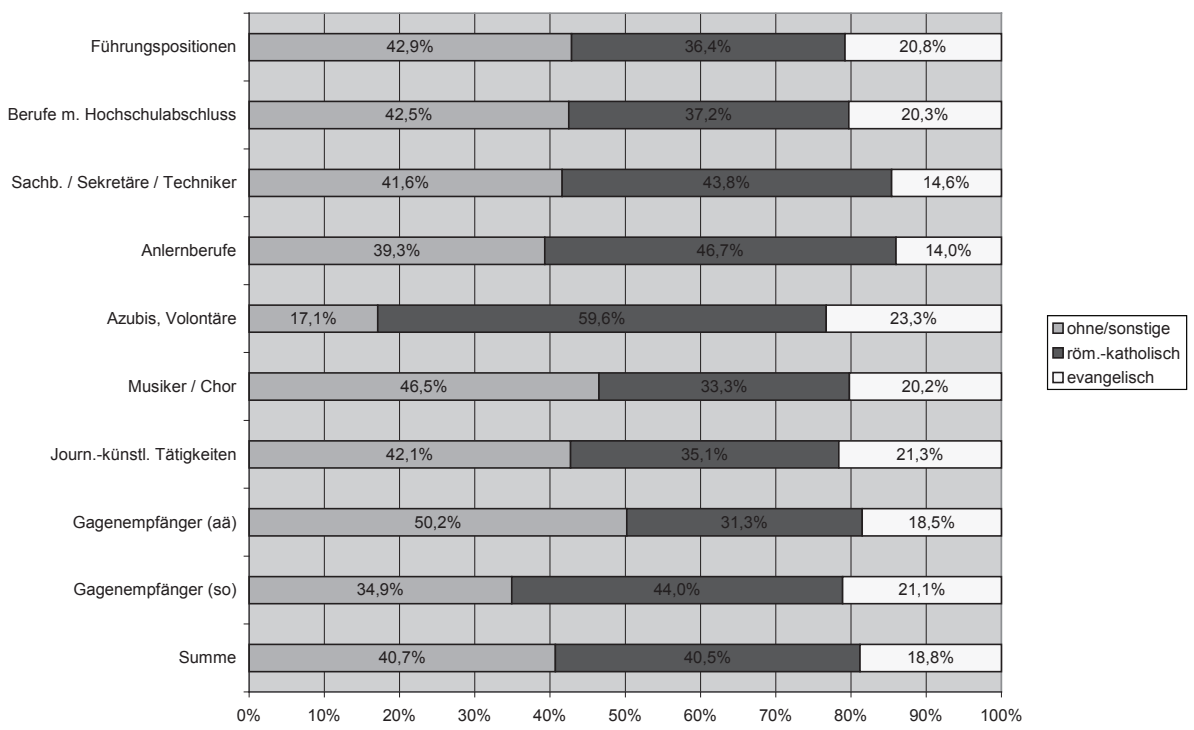

Abb. 6 Konfessionszugehörigkeit der Mitarbeiter des Bayerischen Rundfunks, März 2000

\section{Résumé}

\section{Hierarchie kvalifikace a náboženského vyznání: př́íklad města Mnichova}

Článek předkládá krátký přehled hlavních zájmů autora na poli religiózní geografie. Kromě toho se pokusí zjistit, zda náboženské vyznání může být používáno jako indikátor selektivní mobility vysoce vzdělaných obyvatel. Toto bylo ověřováno na dvou mnichovských univerzitách, u státních úředníkủ v Mnichově (celkem 23000 respondentů), u zaměstnanců největších závodů v průmyslu (BMW a DASA), u komerčních služeb jako např. pojištovnictví a média (Bavorský rozhlas a televize), přičemž do šetření bylo zahrnuto celkem okolo 37000 respondentů. Výzkum prokázal, že čím je vyšší postavení a kvalifikace zaměstnanců, tak tím je i vyšší podíl protestantů (kteří jsou v Bavorsku, resp. v Mnichově v menšině) a také těch, kteří opustili svoji původní církev. Tato skupina je charakteristická opuštěním tradičních vztahů a hodnot a preferuje přitom individuální životní styl.

Prof. emer. Dr. Robert Geipel Institut für Geographie

Ludwig-Maximilians-Universität München

Arcisstr. 21

80333 München

Germany

e-mail: robert.geipel@ssg.uni-muenchen.de,robert.geipel@t-online.de 